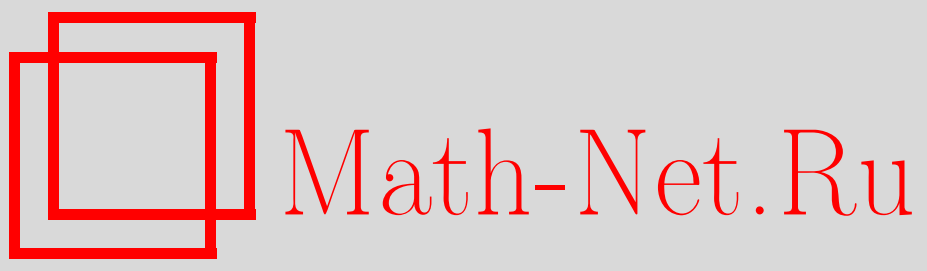

В. В. Рыжиков, Проблема Рохлина о кратном перемешивании в классе действий положительного локального ранга, Функи. анализ и его прил., 2000, том 34, выпуск 1, 90-93

DOI: https://doi.org/10.4213/faa288

Использование Общероссийского математического портала MathNet.Ru подразумевает, что вы прочитали и согласны с пользовательским соглашением

http://www.mathnet.ru/rus/agreement

Параметры загрузки:

IP : 54.162 .127 .20

26 апреля 2023 г., 15:05:52

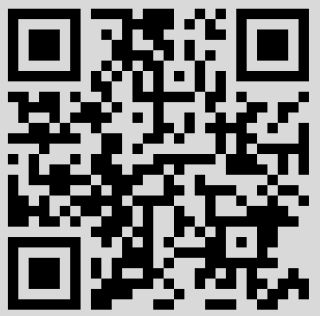


— унитарный оператор; поэтому $\int\left(d \mu_{b}\left(\widetilde{\Phi}^{t}(x)\right) / d \mu_{b}(x)\right) d \mu_{b}(x)=\|V(t) \mathbf{1}\|^{2}=$ $\|\mathbf{1}\|^{2}=1$. Следовательно (см. теорему $4 \S 15$ и замечание к теореме $1 \S 16$ из [9]), $\mu_{b}^{\tilde{\Phi}^{t}} \sim \mu_{b}$ и $\mu_{b}^{\tilde{\Phi}} \sim \mu_{b}$.

Автор выражает благодарность Ю. М. Березанскому за большое внимание к работе и рецензенту за ряд полезных замечаний.

\title{
ЛИТЕРАТУРА
}

1. Dixmier J. Les $C^{*}$-algebras et leur representations, Gautirs-Villars, Paris, 1969. 2. Исмагилов Р. С. Функц. анализ и его прил., 15, вып. 2, 73-74 (1981). 3. Albeverio S., Hoegh-Krohn R., Testard D. J. Funct. Anal., 41, 378-396 (1981). 4. Albeverio S., Hoegh-Krohn R., Testard D., Vershik A. J. Funct. Anal., 51, 115-131 (1983). 5. Kosyak A. V. Selecta. Math. Soviet., 11, 241-291 (1992). 6. Kosyak A. V. J. Funct. Anal., 125, 493-547 (1994). 7. Косяк A. В. Функц. анализ и его прил., 24, вып. 3, 82-83 (1990). 8. Го X.-C. Гауссовы меры в банаховых пространствах. Мир, М., 1976. 9. Скороход A. B. Интегрирование в гильбертовом пространстве. Наука, М., 1975.

\section{Проблема Рохлина о кратном перемешивании в классе действий положительного локального ранга*}

\author{
(c) 2000. В. В. Рыжиков
}

1. В. А. Рохлин [1] ввел в рассмотрение свойство кратного перемешивания и поставил вопрос, влечет ли за собой свойство перемешивания кратности 1 свойства перемешивания кратности $k>1$. Напомним, что автоморфизм $T$ пространства Лебега $(X, \mathscr{B}, \mu), \mu(X)=1$, обладает свойством перемешивания кратности $k$, если для любых множеств $A_{0}, \ldots, A_{k} \in \mathscr{B}$ при $\left|z_{p}-z_{q}\right| \rightarrow \infty$, $0 \leqslant p<q \leqslant k$,

$$
\mu\left(T^{z_{0}} A_{0} \cap T^{z_{1}} A_{1} \cap \cdots \cap T^{z_{k}} A_{k}\right) \longrightarrow \mu\left(A_{0}\right) \mu\left(A_{1}\right) \cdots \mu\left(A_{k}\right) .
$$

Говорят, что автоморфизм $T$ обладает локальным рангом $\beta>0$, если для некоторой последовательности $U_{j}=\bigsqcup_{k \in Q_{j}} T^{z} B_{j}$ башен Рохлина-Халмоша, где $Q_{j}=\left\{0,1, \ldots, h_{j}\right\}$, выполнены следующие условия: $\mu\left(U_{j}\right) \rightarrow \beta$ и для каждого $A \in \mathscr{B}$ пересечение $U_{j} \cap A$ асимптотически близко к объединению некоторого набора этажей в башне. Это означает, что $\mu\left(\left(U_{j} \cap A\right) \Delta \bigsqcup_{z \in S_{j}} T^{z} B_{j}\right) \rightarrow 0$ при $j \rightarrow \infty$ для некоторой последовательности множеств $S_{j} \subset\{0,1, \ldots, h(j)-1\}$ (зависящих от $A)$. Для $\mathbb{Z}^{n}$-действий и $\mathbb{R}^{n}$-действий (потоков) определение аналогично, причем конфигурации $Q_{j}$ имеют вид

$Q_{j}=\left\{0,1, \ldots, h_{1}(j)\right\} \times \cdots \times\left\{0,1, \ldots, h_{n}(j)\right\}, \quad h_{m}(j) \rightarrow \infty, j \rightarrow \infty, 1 \leqslant m \leqslant n$. 
В определении локального ранга потока $\left\{T_{r}\right\}, r \in \mathbb{R}^{n}$, соответствующие башни имеют вид $U_{j}=\bigsqcup_{z \in Q_{j}} T_{t_{j} z} B_{j}$, где $t_{j} \in \mathbb{R}$, причем $t_{j} h_{m}(j) \rightarrow \infty$ при $j \rightarrow \infty$ и $t_{j} \rightarrow 0$ для всех $m, 1 \leqslant m \leqslant n$.

О системах локального положительного ранга см. обзор [3]. Свойство перемешивания кратности 2 для перемешивающих автоморфизмов ранга 1 установил С. Каликов. Его результат стимулировал разнообразные обобщения (см. $[3,5,7])$. Недавно обнаружилась связь проблемы Рохлина с открытыми вопросами спектральной теории. Например, если существует перемешивающий автоморфизм $T$ положительного локального ранга, не обладающий свойством кратного перемешивания, то он имеет фактор с конечнократным абсолютно непрерывным спектром (это вытекает из результатов [4, 6]). Вопрос о реализации такого спектра - давняя задача теории динамических систем (см. обзор [2]). Теперь сформулируем результаты заметки.

ТЕОрема 1. Перемешивающий поток $\left\{T_{r}\right\}, r \in \mathbb{R}^{n}, n \geqslant 1, n p u \beta\left(\left\{T_{r}\right\}\right)>0$ обладает свойством перемешивания всех кратностей.

Теорема 2. Перемешиваюшее действие $\left\{T_{z}\right\}$ групnьı $\mathbb{Z}^{n}, n \geqslant 1, n p u \beta\left(\left\{T_{z}\right\}\right)$ $>1 / 2^{n}$ обладает свойством перемешивания всех кратностей.

2. Дадим набросок доказательства теоремы 1. Ограничимся случаем, когда устанавливается свойство перемешивания кратности 2. Достаточно установить, что $\lambda=\mu \otimes \mu \otimes \mu$ - единственная мера на $X \times X \times X$, удовлетворяющая условиям

$$
\begin{gathered}
\forall r \in \mathbb{R}^{n} \quad \lambda=\left(T_{r} \times T_{r} \times T_{r}\right) \lambda, \\
\int_{X \times X \times X} f \otimes g \otimes h d \lambda=\int f d \mu \int g d \mu \int h d \mu,
\end{gathered}
$$

если одна из функций $f, g, h$ является константой.

Пусть мера $\lambda$ удовлетворяет условиям (1), (2). Определим оператор $J: L_{2}(\mu)$ $\rightarrow L_{2}(\mu \otimes \mu)$ равенством

$$
\int_{X \times X} \chi_{C_{1}} \otimes \chi_{C_{2}} J \chi_{B} d \mu \otimes \mu=\lambda\left(C_{1} \times C_{2} \times B\right) .
$$

Для достаточно большого $N$ рассмотрим следующую меру $\eta$ на $X_{(1)} \times X_{(2)} \times$ $\cdots \times X_{(N+2)}$ :

$$
\begin{aligned}
& \eta\left(C_{1} \times C_{2} \times B_{1} \times \cdots \times B_{N}\right) \\
& \quad=\int_{X \times X} \chi_{C_{1} \times C_{2}}\left(I \otimes T_{\varepsilon}\right) J \chi_{B_{1}} \cdots\left(I \otimes T_{k \varepsilon}\right) J \chi_{B_{k}} \cdots\left(I \otimes T_{N \varepsilon}\right) J \chi_{B_{N}} d \mu \otimes \mu .
\end{aligned}
$$

Если $\lambda \neq \mu \otimes \mu \otimes \mu$, то, как известно, $J^{*} J \neq \Theta(\Theta-$ оператор ортопроекции на пространство констант). Следовательно, полагая $J_{k}=\left(I \otimes T_{k \varepsilon}\right) J$, для достаточно малого $\varepsilon \neq 0$ получаем $J_{h}^{*} J_{k} \neq \Theta$ при $h \neq k$. Оператору $J_{h}^{*} J_{k}$ отвечает мера $\rho$ на $X \times X: \rho(A \times B)=\left\langle J_{h}^{*} J_{k} \chi_{A} \mid \chi_{B}\right\rangle$.

Для любого $\delta>0$ при подходящем выборе $N, h, k$ можно обеспечить свойство $\limsup _{j \rightarrow \infty} \rho\left(U_{j} \times U_{j}\right)=c>\beta^{2}-\delta$. Тогда, рассуждая, как в доказательстве леммы 8 из [7], получим разложение

$$
\rho=c\left(a \int_{\mathbb{R}^{n}}\left(\operatorname{Id} \times T_{v}\right) \Delta d \sigma(v)+(1-a) \mu \otimes \mu\right)+(1-c) \rho^{\prime},
$$


где $\Delta$ - диагональная мера на $X \times X$, а $\sigma$ - некоторая нормированная мера на $\mathbb{R}^{n}$. Случай $a=0$ приводит к $\rho=(1-\delta) \mu \otimes \mu+\ldots$, что при $\delta \rightarrow 0$ влечет за собой равенства $J^{*} J=\Theta, \lambda=\mu \otimes \mu \otimes \mu$. Случай $a>0$ означает, что проекция эргодической компоненты меры $\eta$ на $X_{h} \times X_{k}$ является сдвигом диагональной меры $\left(I \times T_{v}\right) \Delta, v \in \mathbb{R}^{n}$. Последнее приводит к равенству $\left(T_{h \varepsilon} \otimes I \otimes I\right) \lambda=$ $\left(T_{k \varepsilon} \otimes I \otimes T_{v}\right) \lambda$ (здесь мы используем метод доказательства теоремы 1 из [4]). Следовательно, при $v \neq 0$ получим

$\lambda=\left(T_{(h-k) \varepsilon} \otimes I \otimes T_{v}\right) \lambda=\lim _{p \rightarrow \infty}\left(T_{(h-k) p \varepsilon} \otimes I \otimes T_{p v}\right) \lambda=(\Theta \otimes I \otimes \Theta) \lambda=\mu \otimes \mu \otimes \mu$

(здесь мы пользуемся тем, что $T_{u} \rightarrow \Theta$ при $u \rightarrow \infty$, так как поток перемешивает).

При $v=0$ результат не меняется: равенство $\lambda=(\Theta \otimes I \otimes I) \lambda$ влечет за собой $\lambda=\mu \otimes \mu \otimes \mu$. Итак, $\mu \otimes \mu \otimes \mu$ - единственная мера, удовлетворяющая (1), (2), а следовательно, поток обладает свойством перемешивания кратности 2.

3. Чтобы доказать теорему 2, достаточно установить D-свойство рассматриваемых $\mathbb{Z}^{n}$-действий, так как это свойство обеспечивает свойство кратного перемешивания (модификация рассуждений из [7]). Мы говорим, что действие $\left\{T_{z}\right\}$ положительного локального ранга с аппроксимирующей последовательностью $\left(Q_{j}, \xi_{j}, U_{j}\right)$, где $\xi_{j} \rightarrow \varepsilon$ и $\mu\left(U_{j}\right) \rightarrow \beta>0$, обладает D-свойством, если найдется другая аппроксимирующая последовательность $\left(\widetilde{Q}_{j}, \tilde{\xi}_{j}, V_{j}\right)$ и последовательность $\{v(j)\}, v(j) \in \mathbb{Z}^{n}$, такие, что выполнены следующие условия:

(i) $V_{j}=\bigsqcup_{z \in \widetilde{Q}_{j}} T_{z} \widetilde{B}_{j}, \widetilde{Q}_{j} \subset Q_{j}$;

(ii) $V_{j} \subset U_{j}, T_{v(j)} V_{j} \subset U_{j}, \lim _{j \rightarrow \infty} \mu\left(V_{j} \mid U_{j}\right)>0$;

(iii) $\forall z \in \widetilde{Q}_{j} \exists u \in Q_{j} T_{v(j)} T_{z} \widetilde{B}_{j} \subset T_{u} B_{j}$;

(iv) $\max _{z \in \widetilde{Q}_{j}, u \in Q_{j}}\left\{\mu\left(T_{2 v(j)} T_{z} \widetilde{B}_{j} \mid T_{u} B_{j}\right)\right\} \rightarrow 0$.

ТЕОрема 3. Если $\mathbb{Z}^{n}$-действие $\left\{T_{z}\right\}$ обладает свойством перемешивания $u$ $\beta\left\{T_{z}\right\}>1 / 2^{n}$, то это действие обладает D-свойством.

ДокАЗАтельство. Пусть $\left(\widetilde{Q}_{j}, \tilde{\xi}_{j}, U_{j}\right)$ - последовательность башен, которая фигурирует в определении $\beta\left(\left\{T_{z}\right\}\right)$. На каждом шаге с номером $j$ рассмотрим $2^{n}$ множеств вида $T_{\left(w_{1}, w_{1}, \ldots, w_{n}\right)} U_{j}$, где $w_{k} \in\left\{0, h_{k}(j)\right\}$. Taк как $\beta\left(\left\{T_{z}\right\}\right)>1 / 2^{n}$, то при достаточно больших $j$ для различных $\left(w_{1}, \ldots, w_{n}\right)$ и $\left(w_{1}^{\prime}, \ldots, w_{n}^{\prime}\right)$ (которые зависят от $j$ ) имеем

$$
\mu\left(T_{\left(w_{1}, w_{1}, \ldots, w_{n}\right)} U_{j} \cap T_{\left(w_{1}^{\prime}, w_{2}^{\prime}, \ldots, w_{n}^{\prime}\right)} U_{j}\right)=\mu\left(U_{j} \cap T_{\left(w_{1}^{\prime}-w_{1}, \ldots, w_{n}^{\prime}-w_{n}\right)} U_{j}\right)>c
$$

для некоторой константы $c>0$. Не ограничивая общности, предположим, что все величины $w_{k}^{\prime}-w_{k}$ неотрицательны. Общий случай сводится к этому при подходящей замене времени в рассматриваемом действии $\mathbb{Z}^{n}$. При этом роль $B_{j}$ (основания башни) будет играть один из «угловых» этажей башни. Фиксируем маленькое число $\delta>0$ и рассмотрим немного уменьшенную по сравнению с $U_{j}$ башню

$\bar{U}_{j}=\bigsqcup_{z \in \bar{Q}_{j}} T_{z} B_{j}, \quad \bar{Q}_{j}=\left\{0,1, \ldots,\left[(1-\delta) h_{1}(j)\right]\right\} \times \cdots \times\left\{0,1, \ldots,\left[(1-\delta) h_{n}(j)\right]\right\}$

и маленькую башню

$$
\bar{V}_{j}=\bigsqcup_{z \in \delta Q_{j}} T_{z} B_{j}, \quad \delta Q_{j}=\left\{0,1, \ldots,\left[\delta h_{1}(j)\right]\right\} \times \cdots \times\left\{0,1, \ldots,\left[\delta h_{n}(j)\right]\right\}
$$


$([\delta h]$ обозначает целую часть числа $\delta h)$. Из $(3)$ вытекает, что $\mu\left(\bar{U}_{j} \cap T_{2 v(j)} \bar{V}_{j}\right)>$ $a>0$ для некоторой последовательности $\{v(j)\}$, удовлетворяющей условиям $v(j) \in Q_{j}, 2 v(j) \notin Q_{j}$. Отсюда мы получим, что $\mu\left(U_{j} \mid T_{2 v(j)} B_{j}\right)>a$; следовательно, положив $\widetilde{B}_{j}=T_{-2 v(j)} U_{j} \cap B_{j}$, мы определим башню $V_{j}=\bigsqcup_{z \in \widetilde{Q}_{j}} T_{z} \widetilde{B}_{j}$. Условия (i), (ii), (iii) выполнены по построению. Условие (iv) вытекает из свойства перемешивания (см. доказательство утверждения 7 из [7]).

Автор обязан Б. М. Гуревичу, В. И. Оселедцу, А. М. Степину и Ж.-П. Тувено за интерес к результатам и полезные обсуждения.

\title{
ЛИТЕРАТУРА
}

1. Рохлин B. А. Известия АН СССР, сер. матем., 13, 329-340 (1949). 2. Вершик А. М., Корнфельд И. П., Синай Я. Г. В кн.: Динамические системы-2. Совр. проблемы математики. Фундаментальные направления. Итоги науки и техники, Т. 2, ВИНИТИ, М., 1985, с. 5-111. 3. Ferenczi S. Colloq. Math., 73, No. 1, 35-65 (1997). 4. Glasner E., Host B., Rudolph D. Israel J. Math., 78, 131-142 (1992). 5. del Junco A., Yassawi $R$. Multiple mixing and rank one group actions (to appear in Canad. J. Math.). 6. King J., Thouvenot J.-P. J. Anal. Math., 56, 211-230, (1991). 7. Рьюиков В. В. Функц. анализ и его прил., 27, No. 2, 63-78 (1993).

Московский государственный университет им. М. В. Ломоносова, Поступило в редакцию механико-математический факультет 18 февраля 1998 г.

\section{УДК 517.986}

\section{Вещественные факторы типа III $_{0}$}

\author{
(C) 2000. Ш. М. УСмАнов
}

Вещественной $W^{*}$-алгеброй называется вещественная *-алгебра $R$ ограниченных линейных операторов в комплексном гильбертовом пространстве $H$, содержащая единицу, замкнутая в слабой операторной топологии и такая, что $R \cap i R=\{0\}$. В работе получено дискретное разложение $\sigma$-конечных вещественных факторов типа $\mathrm{III}_{0}$ (cp. [1]).

1. Предварительные сведения. Пусть $R$ - вещественная $W^{*}$-алгебра. Напомним, что обертываюшая $W^{*}$-алгебра $\mathscr{U}(R)$ для $R$ (т.е. наименьшая $W^{*}$-алгебра, содержащая $R$ ) имеет вид $\mathscr{U}(R)=R+i R$ и $R$ определяет на $\mathscr{U}(R)$ некоторый *-антиавтоморфизм $\alpha_{R}$ периода $2: \alpha_{R}(x+i y)=x^{*}+i y^{*}$, где $x, y \in R$. Соответственно и $R$ определяется при помощи $\alpha_{R}: R=\{x \in \mathscr{U}(R)$ : $\left.\alpha_{R}(x)=x^{*}\right\}$. Вещественные $W^{*}$-алгебры $R$ и $Q$, порождающие одну и ту же $W^{*}$-алгебру $\mathscr{U}, \mathscr{U}(Q)=\mathscr{U}(R)=\mathscr{U},{ }^{*}$-изоморфны тогда и только тогда, когда $\alpha_{R}$ и $\alpha_{Q}$ на $\mathscr{U}$ сопряжены, т.е. существует ${ }^{*}$-автоморфизм $\theta \in$ Aut $\mathscr{U}$, такой, что $\alpha_{R}=\theta \cdot \alpha_{Q} \cdot \theta^{-1}$ (см. [2,3]). Пусть $R$ и $Q$ являются *-изоморфными и, следовательно, $M=\mathscr{U}(R)$ и $N=\mathscr{U}(Q)$ комплексно *-изоморфны и антиавтоморфизмы $\alpha_{R}$ и $\alpha_{Q}$ сопряжены с помощью этого изоморфизма; тогда будем говорить, что пары $\left(M, \alpha_{R}\right)$ и $\left(N, \alpha_{Q}\right)$ сопряжены. Вещественная *-алгебра $R$ называется вещественным фактором, если ее центр тривиален, $Z(R)=\{\mathbb{C} 1\}$ (и тогда $\mathscr{U}(R)$ - комплексный фактор); $R$ имеет тип $\mathrm{I}_{\mathrm{fin}}\left(\mathrm{I}_{\infty}, \mathrm{II}_{1}, \mathrm{II}_{\infty}, \mathrm{III}_{\lambda}\right.$, 